\title{
Development of Performance Deterioration Diagnosis Method for Gas Turbine Combined Cycle Power Plants*
}

\author{
Toru TAKAHASHI**, Eiichi KODA** and Yoshinobu NAKAO** \\ ${ }^{* *}$ Central Research Institute of Electric Power Industry, \\ 2-6-1 Nagasaka, Yokosuka-shi, Kanagawa-ken 240-0196, Japan \\ E-mail: toru-tak@criepi.denken.or.jp
}

\begin{abstract}
It is necessary to maintain or improve the thermal efficiency of actual thermal power generation plants from the viewpoint of environmental problems. When the thermal efficiency decreases, it is crucial to identify the performance deterioration factors by appropriate energy management. However, it is difficult to understand the internal state of such plants sufficiently and to determine performance deterioration factors only from operation data. In particular, investigating the performance deterioration factors is very difficult in gas turbine combined cycle (GTCC) power plants because the performance changes markedly depending on atmospheric conditions. Therefore, we develop a method based on heat and mass balance analysis to calculate the immeasurable quantity of state and the component performance values in GTCC power plants, and to correct them to the values under standard conditions using the performance function obtained from long-term operation data. From the results, we can identify the main factors affecting the thermal efficiency deterioration of GTCC power plants using this method.
\end{abstract}

Key words: Gas Turbine Combined Cycle, Power Plant, Thermal Efficiency, Performance Deterioration, Heat and Mass Balance Analysis

\section{Introduction}

In order to prevent the increase in $\mathrm{CO}_{2}$ emission and energy consumption worldwide, it is necessary to maintain or improve the thermal efficiency of actual thermal power generation plants. It is also important to reduce the maintenance cost of commercial thermal power plants. When the thermal efficiency decreases, it is crucial to identify the performance deterioration factors by appropriate energy management and solve the problem immediately. The heat loss method or heat input-output method is usually used for energy management in actual thermal power plants [1], [2]; however, even with the use of these methods, when the plant thermal efficiency decreases, it is still difficult to determine the main cause of such a decrease from among several components of a power plant. In addition, it is more difficult to manage plant thermal efficiency sufficiently in gas turbine combined cycle (GTCC) power plants because plant performance itself changes markedly depending on external conditions such as atmospheric temperature and seawater temperature. Thus, establishing a performance deterioration diagnosis method is very important for maintaining the performance of actual GTCC power plants. However, there have been few studies from this viewpoint.

We have developed an algorithm that can analyze the heat balance of various power generation systems [3]. Software with a graphic user interface has also been developed by 
applying the algorithm [4]. The algorithm and software have been used for the analysis, comparative estimation, and optimization of some new high-efficiency generation systems [5], [6]. We have also analyzed the heat balance of various existing steam power plants and developed a method of determining the factors affecting performance deterioration in steam power plants with reduced plant thermal efficiency [7]. However, there has been no such method for analyzing GTCC power plants which consist of many components and are markedly affected by changes in external factors; thus such plants have not been sufficiently analyzed.

Therefore, we develop a performance deterioration diagnosis method of determining the factors affecting the thermal efficiency of GTCC power plants by excluding external conditions such as ambient condition changes.

In this paper, we introduce the developed method and discuss an actual study of identifying the deterioration factors for a 250MW-class GTCC power plant.

\section{Nomenclature}

\section{Symbol Definitions}

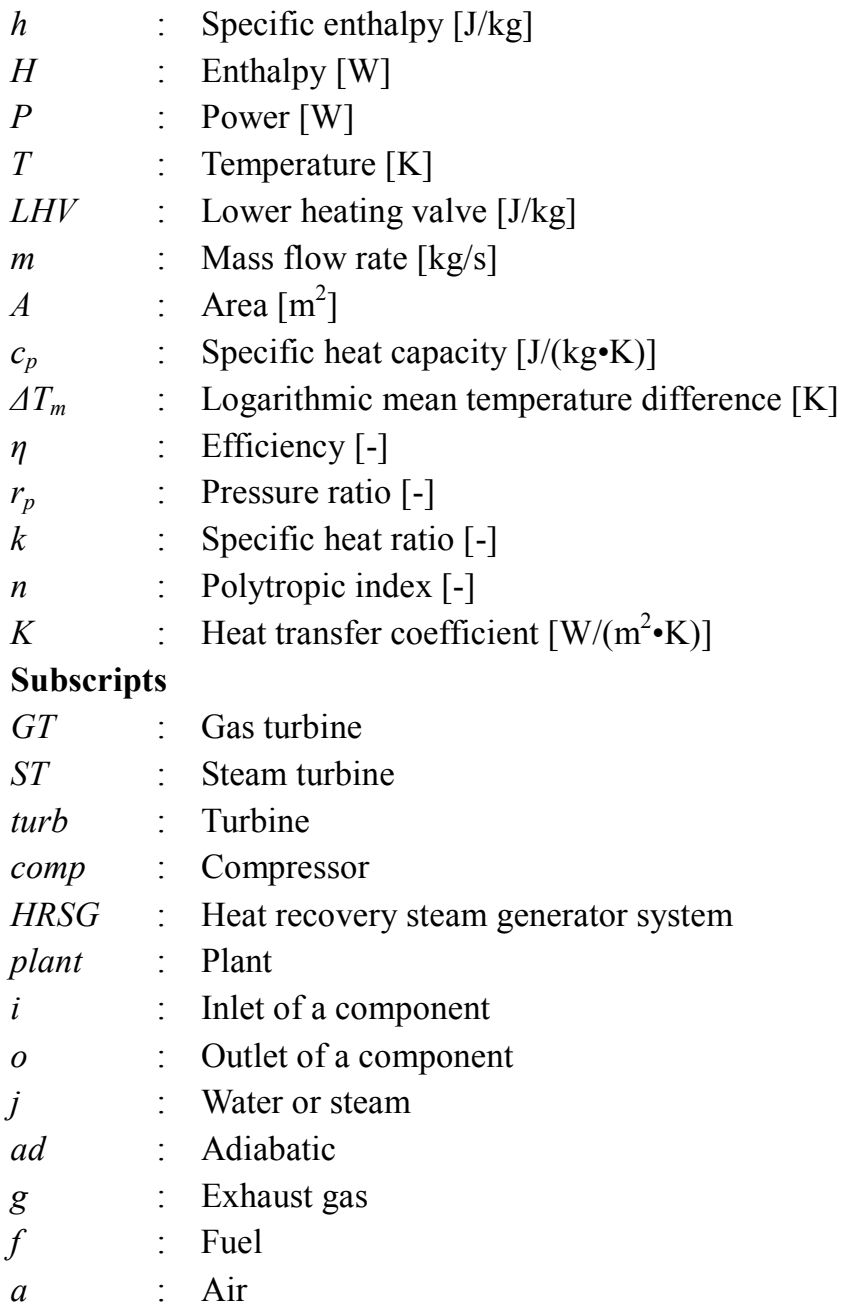

\section{Problem and solution}

\subsection{Problem with current thermal efficiency management in GTCC power plants}

Figure 1 shows a schematic diagram of a commercial GTCC power plant [8]. It generally consists of many pieces of machinery and equipment such as a gas turbine (GT), a 
compressor (COMP), a high-pressure steam turbine (HPT), an intermediate- and low-pressure steam turbine (ILPT) and a heat recovery steam generator system (HRSG) composed of many heat exchangers.

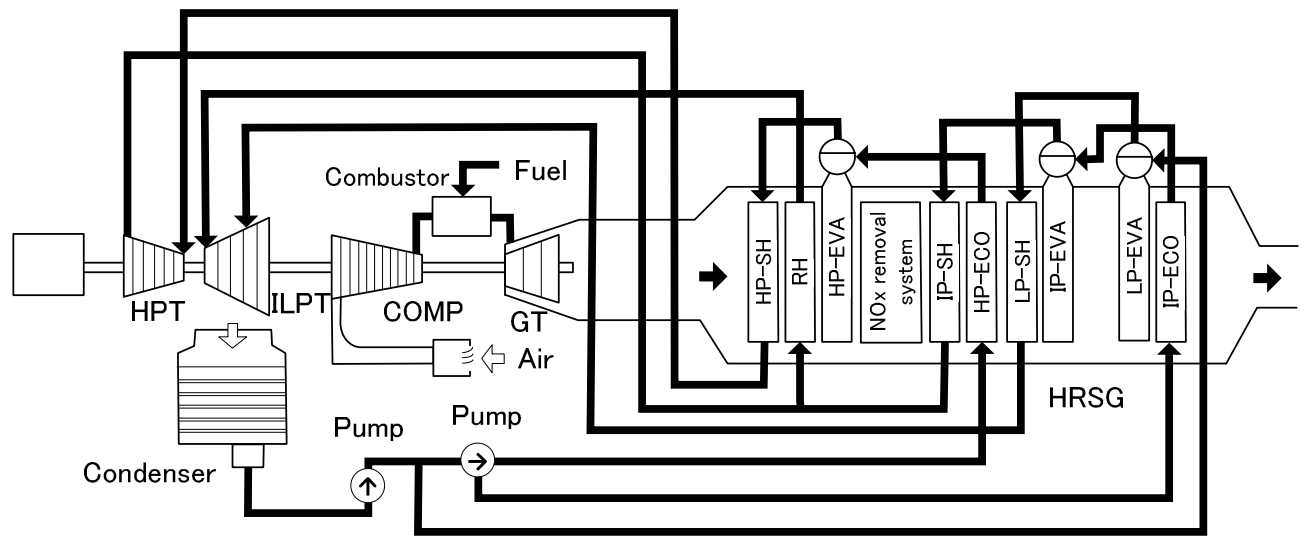

Fig. 1 Schematic diagram of a GTCC power plant.

The thermal efficiency of GTCC power plants is managed by adjusting indices such as GT thermal efficiency $\eta_{G T}$, ST thermal efficiency $\eta_{S T}$ and HRSG thermal efficiency $\eta_{H R S G}$, aside from plant thermal efficiency $\eta_{\text {plant }}$ which reflects the thermal efficiency of the entire plant. The heat input-output method is used and the expressions for these indices are defined as follows.

$$
\begin{aligned}
& \eta_{G T}=\frac{P_{G T}}{H_{f}+H_{a}+m_{f} \cdot L H V}=1-\frac{H_{g}}{H_{f}+H_{a}+m_{f} \cdot L H V} \\
& \eta_{S T}=\frac{P_{S T}}{\sum\left(m_{j} \Delta h_{j}\right)} \\
& \eta_{H R S G}=\frac{\sum\left(m_{j} \Delta h_{j}\right)}{H_{g}} \\
& \eta_{\text {plant }}=\frac{P_{\text {plant }}}{H_{f}+H_{a}+m_{f} \cdot L H V}
\end{aligned}
$$

As there is a relationship between the powers of Plant, GT and ST, $P_{\text {plant }}, P_{G T}$ and $P_{S T}$, as shown in Eq. (5), Eq. (6) is obtained from Eqs. (1) to (5).

$$
\begin{aligned}
& P_{\text {plant }}=P_{G T}+P_{S T} \\
& \eta_{\text {plant }}=\left(1-\eta_{G T}\right) \cdot \eta_{S T} \cdot \eta_{H R S G}+\eta_{G T}
\end{aligned}
$$

From these equations, when the plant thermal efficiency decreases, it can be understood that its cause is related to GT, ST or HRSG. However, even with the use of these indices and equations, it is still difficult to identify the cause of such a decrease from among several components of a GTCC power plant.

In particular, in the case of single-shaft GTCC power plants, it is difficult even to obtain $P_{G T}$ and $P_{S T}$, individually. Although a new method using an optical torque sensor has been reported by Umezawa [9], $P_{G T}$ and $P_{S T}$ are usually estimated from a function of feed water flow rate or air flow rate at present. Therefore, if the performance of the plant changes, each 
value obtained using these functions cannot correspond to the actual power. From the results, these efficiencies calculated using the above equations could also be different from the actual value.

Moreover, it is difficult to manage plant thermal efficiency effectively because plant performance changes markedly depending on external conditions such as atmospheric temperature and seawater temperature in GTCC power plants. When the component performance changes depending on these external conditions, even determining the performance change of the component itself becomes difficult.

\subsection{Development of Performance Deterioration Diagnosis Method}

To solve the problem shown in the previous section, a performance deterioration diagnosis method of identifying the factors causing the decrease in the thermal efficiency of GTCC power plants is developed. Fig 2 shows the procedure for identifying the cause of thermal efficiency deterioration. The main steps of the procedure are as follows.

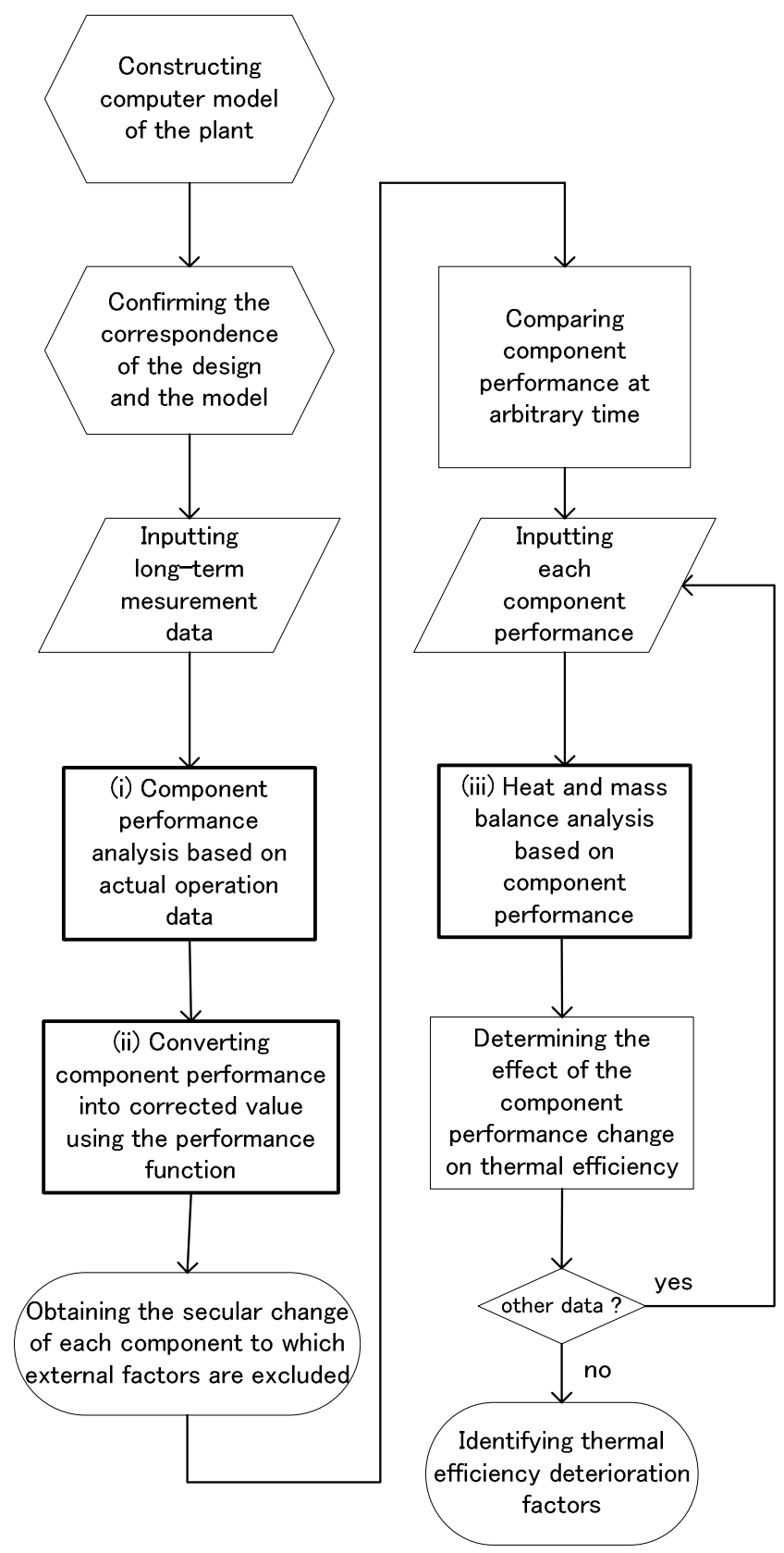

Fig. 2 Procedure for analyzing performance deterioration factors. 
i. The GTCC power plant model based on heat and mass balance is constructed using the program. Measurement points are confirmed and data input points are matched to them.

ii. The correspondence of the design heat and mass balance with the analysis model calculation value is confirmed to verify the validity of the constructed model.

iii. The heat and mass balance is analyzed using long-term operation data, and the secular change of the performance of each component is understood from these results.

iv. The correlations of entry conditions and component performance are investigated for each component, and performance functions of each component are created.

v. Component performance is converted into that under standard conditions using the performance function.

vi. The thermal efficiency and performance of each component change at arbitrary times, for example, at the start of commercial operation and at present, are quantitatively compared.

vii. The effect of a change in each component performance on the thermal efficiency of the plant is calculated.

viii. The main thermal efficiency deterioration factors are determined.

The parts enclosed by a bold frame in this figure are the most important in this method. Each part is explained as follows.

\section{(i) Component performance analysis based on actual operation data}

It is thought that the cause of the plant thermal efficiency decrease can be deduced if each performance factor of individual components such as the adiabatic efficiency of COMP $\eta_{a d, \text { comp }}$ shown in Eq. (7), that of turbines such as GT and ST $\eta_{a d, \text { turb }}$ shown in Eq. (8) and the heat transfer coefficient $K$ shown in Eq. (9) of each heat transfer surface in HRSG can be obtained.

$$
\begin{aligned}
& \eta_{a d, \text { comp }}=\frac{h_{a d}-h_{i}}{h_{o}-h_{i}}=\frac{c_{p, a d} \cdot T_{a d}-c_{p, i} \cdot T_{i}}{c_{p, o} \cdot T_{o}-c_{p, i} \cdot T_{i}} \\
& \eta_{a d, t u r b}=\frac{h_{i}-h_{o}}{h_{i}-h_{a d}}=\frac{c_{p, i} \cdot T_{i}-c_{p, o} \cdot T_{o}}{c_{p, i} \cdot T_{i}-c_{p, a d} \cdot T_{a d}} \\
& K=\frac{\Delta H_{j}}{A \cdot \Delta T_{m}}=\frac{m_{j} \cdot c_{p} \cdot\left(T_{j, o}-T_{j, i}\right)}{A \cdot\left(\left(T_{g, i}-T_{j, o}\right)-\left(T_{g, o}-T_{j, i}\right)\right)} \cdot \ln \frac{\left(T_{g, i}-T_{j, o}\right)}{\left(T_{g, o}-T_{j, i}\right)}
\end{aligned}
$$

The quantity of state of the working fluid at the inlet and outlet of each component is required to calculate the above performance factors, but every data point cannot necessarily be measured. For instance, it is difficult to measure combustion temperature directly because it is very high. Also, part of the steam at the ILPT exit condenses and the wetness fraction cannot be determined only by temperature and pressure; therefore, the adiabatic efficiency cannot be calculated directly using the measurement value because the enthalpy is uncertain.

Thus, by analyzing the heat and mass balance from the measured operation data, it is possible to determine the quantity of state in the part that cannot be measured and the performance value of each component. Figure 3 shows the procedure for the component performance analysis based on actual operation data. 


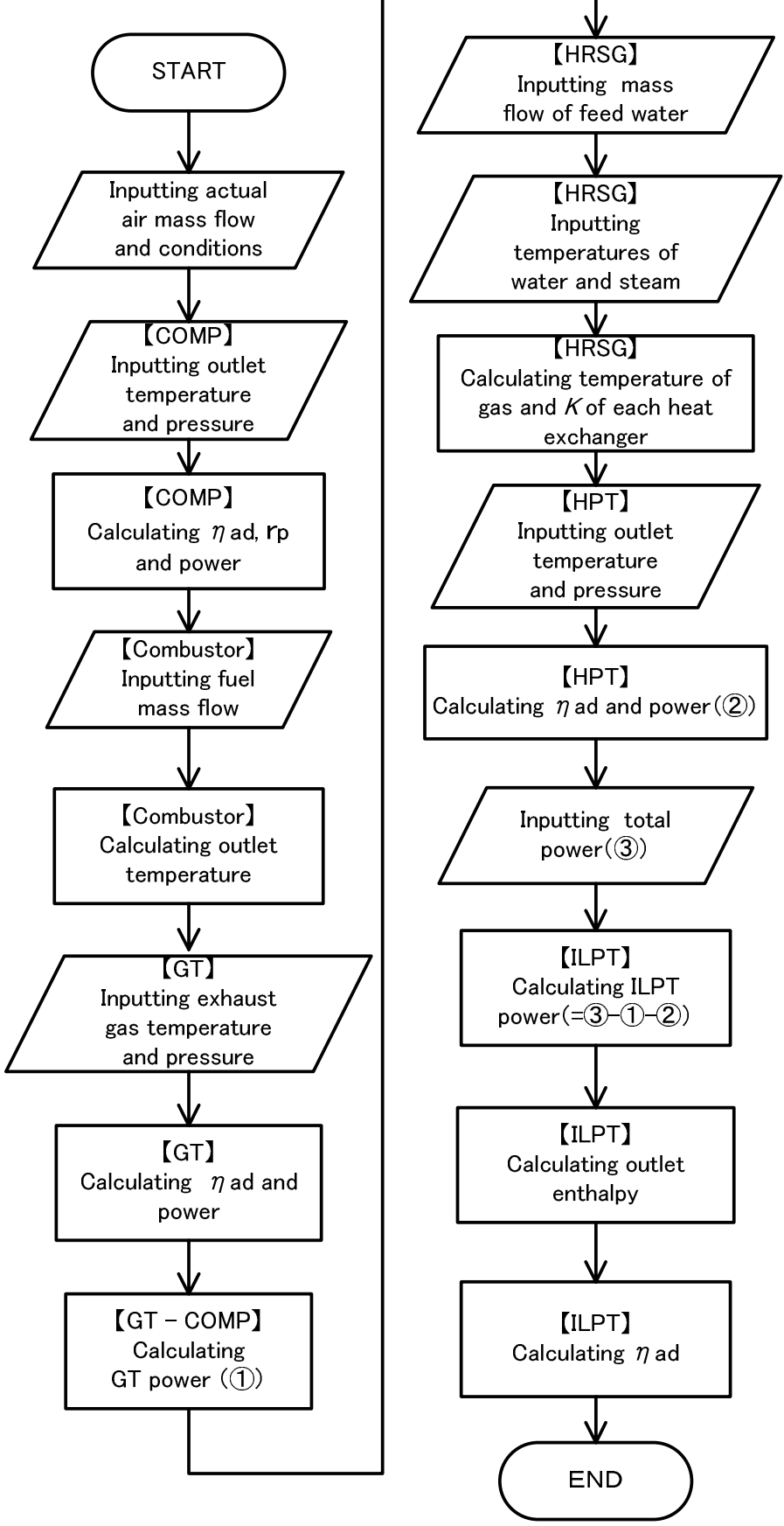

Fig. 3 Procedure of component performance analysis based on actual operation data.

As the inside of the plant continually changes with the disorder in the flow and turbulence even in the stationary state of constant load, and the quantity of state is not necessarily constant, the performance change is not correctly evaluated using only a certain instantaneous value. Therefore, it is considered necessary to evaluate the secular change in each component performance by processing a huge amount of data collected over a long term at short intervals.

Moreover, there is a possibility that the accuracy of some values such as combustion temperature and enthalpy of ILPT exit that are obtained from the calculation using some measurement values is getting worse because the error margins of the measurement values are accumulated. Thus, it is necessary to improve the accuracy by averaging a lot of data at short intervals or by extracting data with high reliability based on probability statistics. 
(ii) Converting component performance into corrected value using the performance function

In some components, the performance itself changes depending on external conditions such as atmospheric conditions and the seawater temperature even at a constant load. To understand the performance without the effect of external conditions, it is necessary to convert component performance into the corrected value. Thus, it is considered that the conversion is possible by designing performance functions whose autonomous variable are the quantity of state of the working fluid at the component inlet and substituting the value of the standard condition as the autonomous variable. Figure 4 shows the concept of converting component performance into its corrected value using performance functions. It is assumed that the component performance calculated from the actual operation data at a certain time was point A and that calculated at another time was point B. Although these are almost the same, the conditions of the working fluid are markedly different. The quadratic coefficient and linear coefficient of the performance functions with respect to the working fluid condition are determined by fitting long-term operation data by the least-squares method or other methods. The constant term of the performance function at each point is determined by fitting the point by the parallel displacement of the performance function with the quadratic coefficient and linear coefficient.

Thus, the quadratic coefficient and linear coefficient of the performance function show the characteristic of the component, and the difference in the constant term of the performance function shows the change in the performance of the component. It is possible to convert them into the corrected values under the standard condition using the function. As a result, the difference in the corrected values can be considered as deterioration in performance of the component.

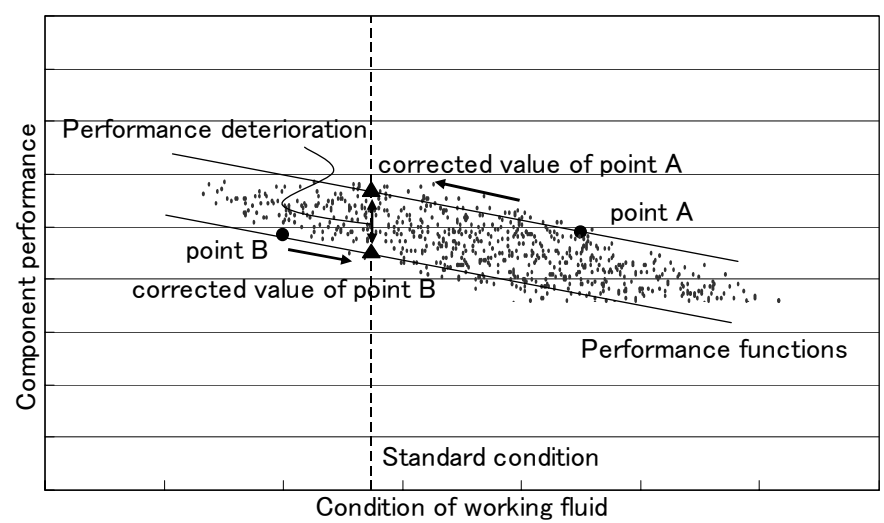

Fig. 4 Conceptual diagram of converting component performance into the corrected value using the performance function.

\section{(iii) Heat and mass balance analysis based on component performance}

The components whose performance has changed can be found by the above-mentioned method. However, the component whose performance has decreased most is not necessarily the main factor causing the thermal efficiency decrease of the plant. This is because the effect of the component performance change on plant thermal efficiency differs from one component to another. For example, if the adiabatic efficiency of COMP, GT, HPT and ILPT decreases by $1 \%$, the effect of these changes on plant thermal efficiency differs depending on the component because power differs depending on the component. Moreover, the performance indices differ depending on the component.

In order to determine the main factor causing the thermal efficiency decrease, it is considered effective to estimate the effects of these performance deterioration factors on thermal efficiency by heat and mass balance analysis based on component performance. 
Figure 5 shows the procedure used for the calculation. By repeating this procedure for each change in component performance value as shown in Fig 2, it should be possible to understand quantitatively how much the performance change of each individual component affects the thermal efficiency.

From the results, it is considered possible to identify the main cause of plant performance deterioration.

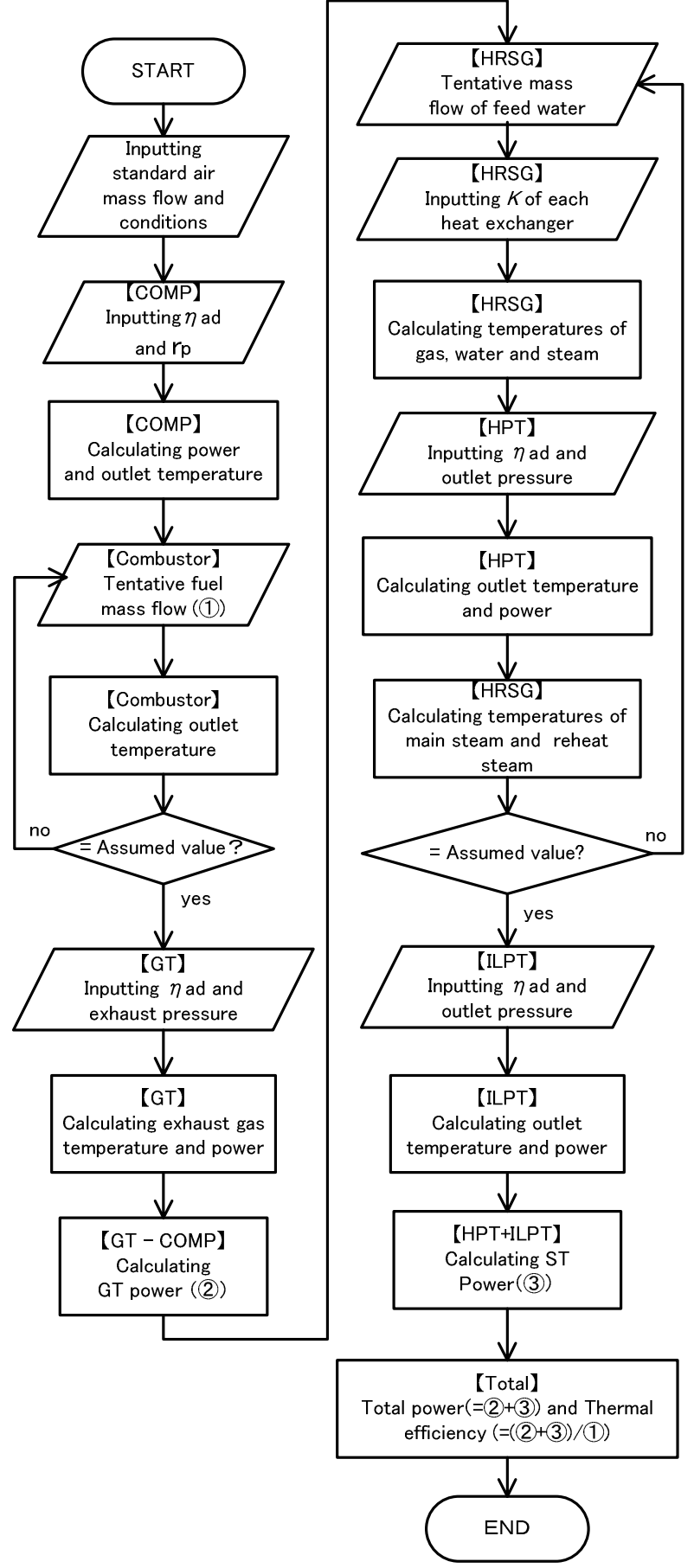

Fig. 5 Procedure of heat and mass balance analysis based on component performance.

By analyzing the effect of each factor on thermal efficiency quantitatively, economic evaluation also becomes possible. That is, the factor that most strongly determines cost-effectiveness can be understood by calculating and comparing the decrease in running cost due to fuel reduction with that in repair cost due to component improvement. 


\section{Analysis results}

\subsection{Investigation of factors that affect thermal efficiency}

A single-shaft 250MW-class GTCC power plant is analyzed using the proposed method. The thermal efficiency of this plant decreased by $1.4 \%$ within about four years from the performance test conducted immediately after the fast periodic inspection (fast performance test) to the performance test conducted immediately after the fourth periodic inspection (fourth performance test); thus, it is necessary to ascertain the cause of performance deterioration.

The heat balance of this plant is analyzed using the measurement operation data, and the performance of each component is calculated; the secular change in the performance of each component is also investigated. Moreover, the main measurement data such as main and reheat steam temperatures and pressures that markedly affect thermal efficiency are also investigated. The operation data from IGV $86 \%$ to $\max 87 \%$ every hour for about four years is extracted as input data for this analysis to exclude the effect of the change in the volume flow of the inlet air into the compressor on plant performance.

As the performance of some components might be affected by external factors, the following examinations are conducted. Figures 6 and 7 respectively show the secular change of adiabatic efficiency and pressure ratio of the compressor calculated from the actual measurement operation data. From these figures, it is difficult to understand the performance change of the compressor itself because both parameters change with the season. Adiabatic efficiency tends to be higher in summer and lower in winter; on the other hand, pressure ratio tends to be higher in winter and lower in summer. Therefore, it is necessary to exclude the effect of seasonal changes, that is, changes in the ambient condition to understand the performance change of the compressor itself.

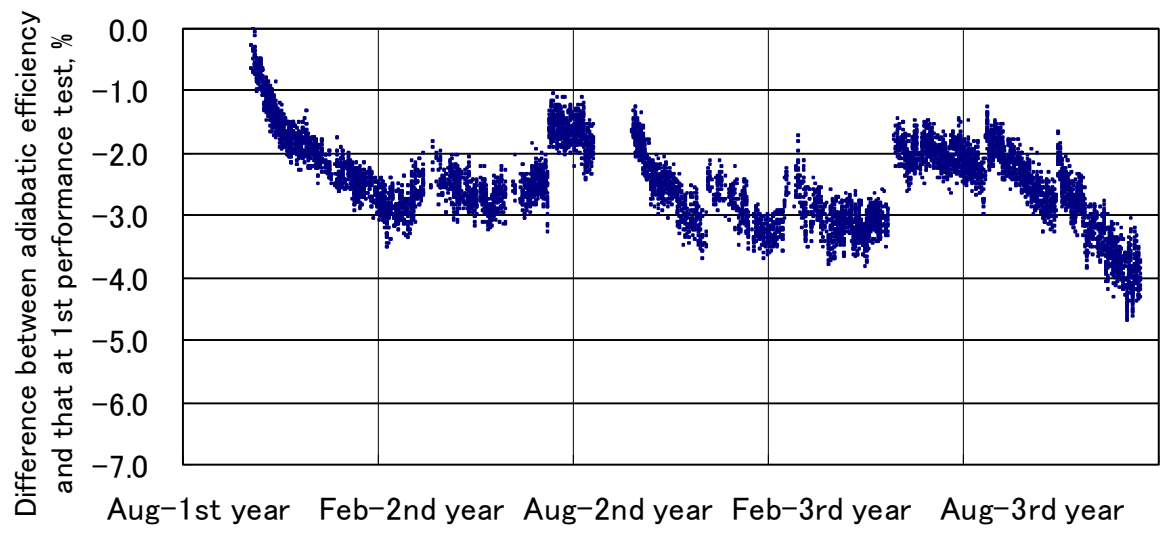

Fig. 6 Secular change of adiabatic efficiency of compressor.

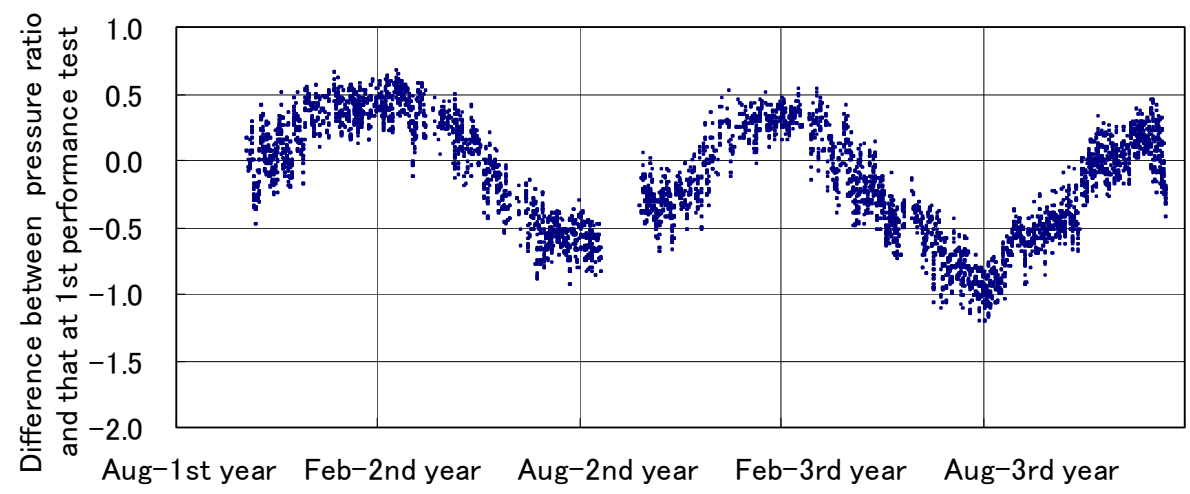

Fig. 7 Secular change of pressure ratio of compressor. 
The correlations of each of the above two performance values with ambient conditions such as temperature, pressure, relative humidity and the mass flow rate of compressor inlet air are examined. It is found that both performance values have very good correlations with the mass flow rate compared with other parameters, as shown in Figs 8 and 9, respectively. Since atmospheric temperature, pressure and relative humidity change with the season and the weather, air density changes. As a result, the mass flow rate changes with the season and the weather when the volume flow of compressor inlet air is constant.

It has been reported that pressure ratio changes with the mass flow rate [10]. Moreover, the adiabatic efficiency of compressor is shown in Eq. (10) using the pressure ratio $r_{p}$ from Eq. (7) when specific heat capacity of the working fluid at compressor inlet and that at its exit is considered to be almost the same.

$$
\eta_{a d, c o m p}=\frac{T_{a d} / T_{i}-1}{T_{o} / T_{i}-1}=\frac{r_{p}^{\frac{\kappa-1}{\kappa}}-1}{r_{p}^{\frac{n-1}{n}}-1}
$$

As shown in Eq. (10), adiabatic efficiency depends on pressure ratio; therefore, it is theoretically appropriate that adiabatic efficiency is also obtained as a function of mass flow rate.

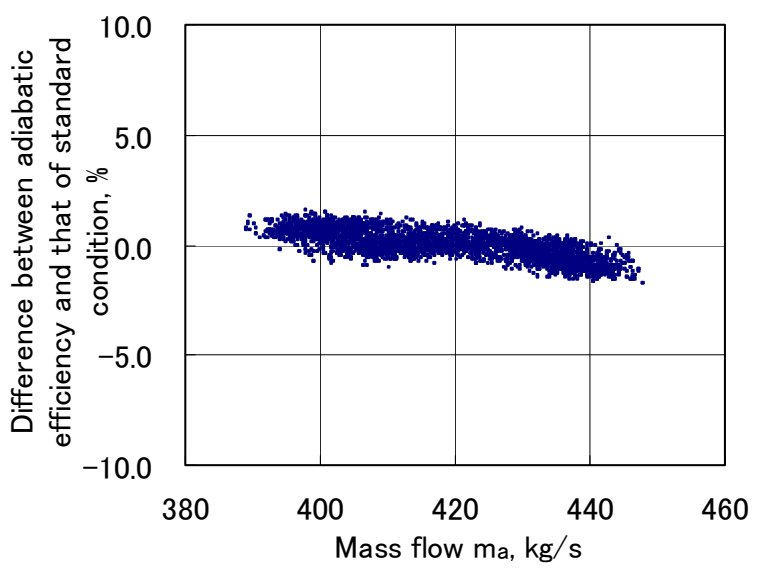

Fig. 8 Relationship between adiabatic efficiency of compressor and air mass flow rate

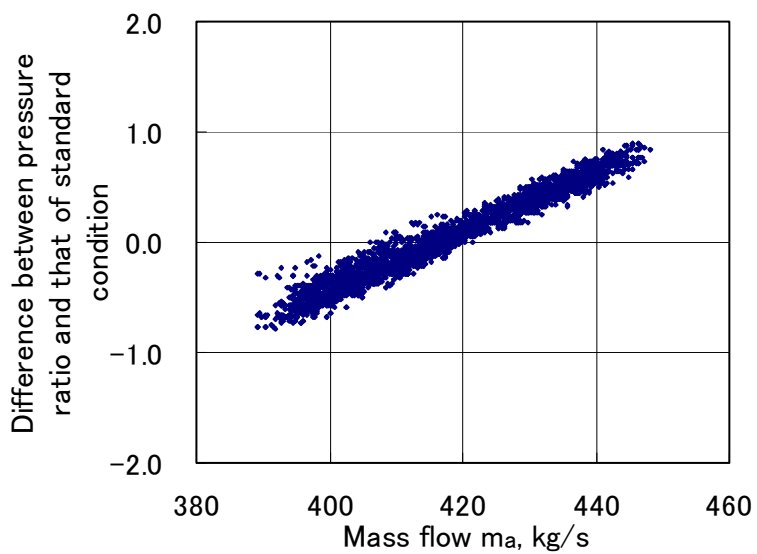

Fig. 9 Relationship between pressure ratio of compressor and air mass flow rate.

The performance functions at each point in Figs 8 and 9 are obtained as follows. The quadratic coefficient and linear coefficient of each performance function with respect to the mass flow rate $m_{a}$ are determined by fitting the data obtained from long-term measurement 
to a quadratic curve by the least-squares method. In this case, Eqs. (11) and (12) are respectively obtained as the performance functions of adiabatic efficiency and pressure ratio.

$$
\begin{aligned}
& \eta_{a d, \text { comp }}=-3.98 \cdot 10^{-4} \cdot m_{a}{ }^{2}+3.02 \cdot 10^{-1} \cdot m_{a}+c_{1} \\
& r_{p}=1.13 \cdot 10^{-5} \cdot m_{a}{ }^{2}+1.74 \cdot 10^{-2} \cdot m_{a}+c_{2}
\end{aligned}
$$

The constant terms $c_{1}$ and $c_{2}$ of the performance functions at each point are determined by fitting the point by the parallel displacement of the performance function with the quadratic coefficient and linear coefficient. Therefore, the corrected adiabatic efficiency and pressure ratio are obtained from the standard mass flow rate.

Figures 10 and 11 respectively show the results indicating that adiabatic efficiency and pressure ratio calculated from the actual measurement operation data are respectively corrected by Eqs. (11) and (12) when the standard atmospheric conditions for the performance function are $15^{\circ} \mathrm{C}, 1013 \mathrm{kPa}$ and $\mathrm{RH} 70 \%$.

As the figures show, the performance changes caused by the ambient conditions observed in Figs 6 and 7 can be excluded. Therefore, the performance change of the compressor itself can be observed clearly compared with those shown in Figs 6 and 7. Thus, it is clear that adiabatic efficiency and pressure ratio recover with blade washing and maintenance, but decrease gradually over a long time in the GTCC power plant studied.

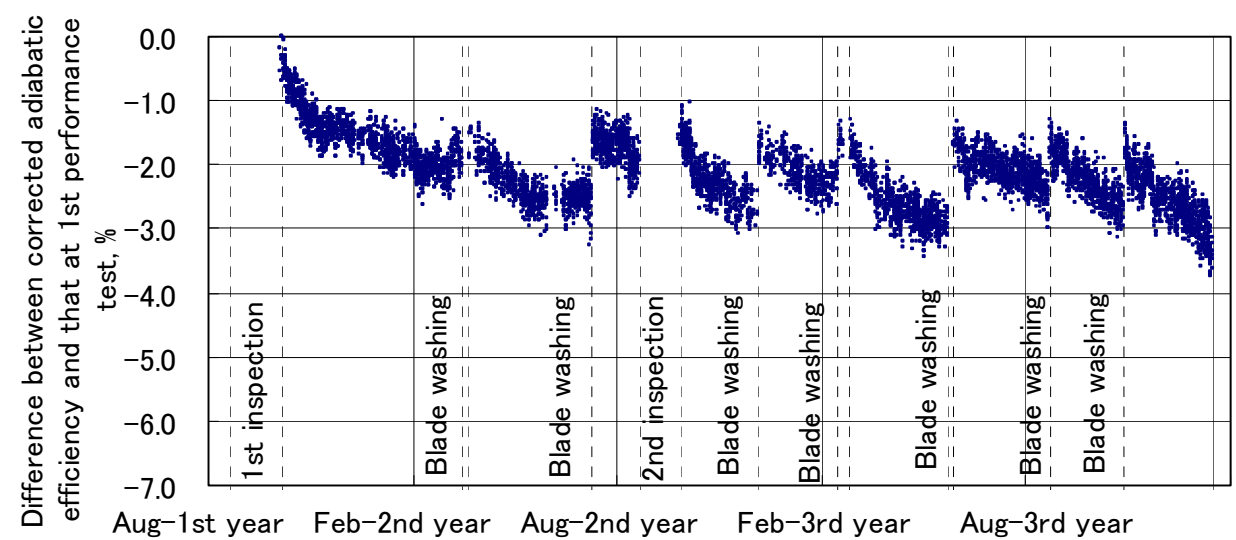

Fig. 10 Secular change of the corrected adiabatic efficiency of compressor.

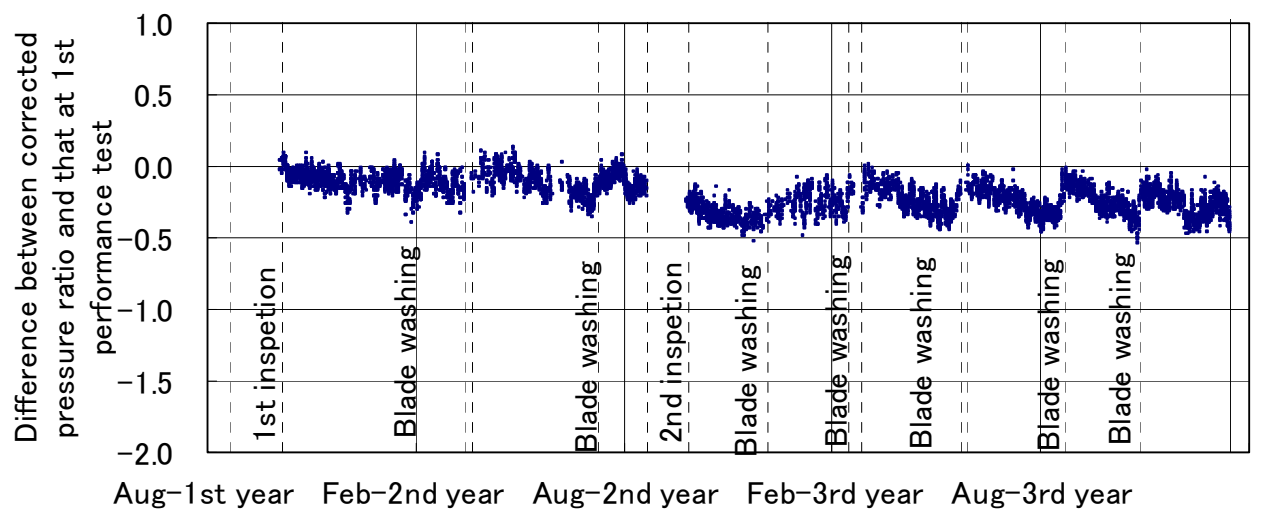

Fig. 11 Secular change of the corrected pressure ratio of compressor. 
From the results of similarly examining the other components, it is found that the vacuum of the condenser correlates well with seawater temperature, and GT exhaust gas pressure correlates well with the mass flow rate of the exhaust gas. These relationships can also be expressed with each performance function; therefore, it is possible using the performance functions to exclude the effect of season on performance and clarify the performance deterioration itself.

The performance of each component in the fourth performance test is compared with that in the first performance test; both results are corrected to the standard atmospheric conditions and seawater temperature $\left(22^{\circ} \mathrm{C}\right)$ using the above-mentioned method. The results are shown in Figs 12 and 13. From the figures, the thermal efficiency deterioration factors are as follows.

- HPT adiabatic efficiency decrease

- ILPT adiabatic efficiency decrease

- Compressor adiabatic efficiency and pressure ratio decrease

- Heat transfer performance deterioration of HRSG (In Fig 13, HP, IP and LP denote high, intermediate and low pressures, respectively; SH, EVA and ECO denote superheater, evaporator and economizer, respectively)

- Condenser vacuum decrease

- Heat loss increase of main steam pipe

- GT exhaust gas pressure increase (HRSG differential pressure increase)

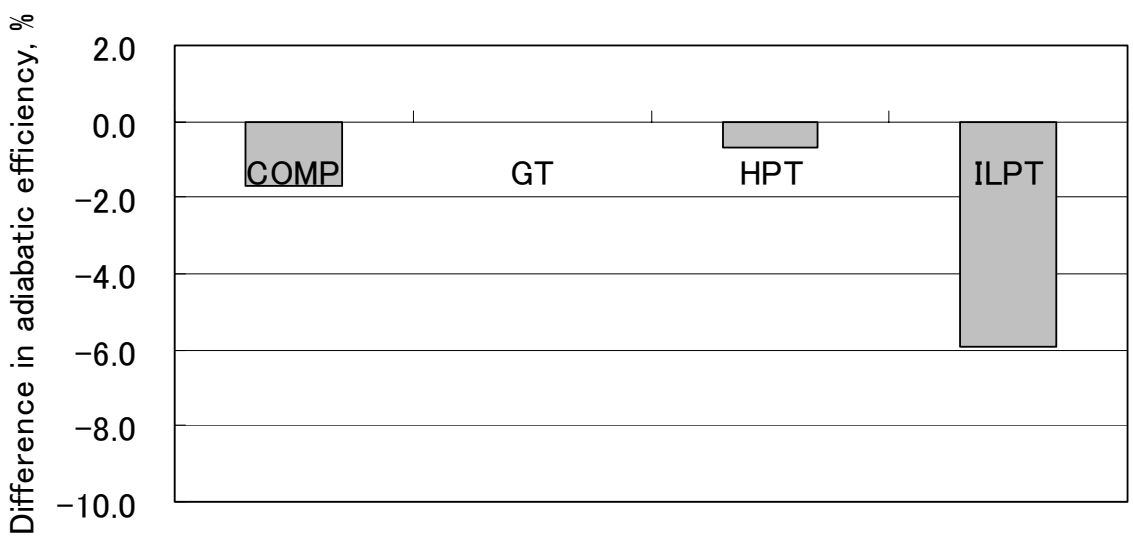

Fig. 12 Change in adiabatic efficiency of each rotary machine over about 4 years.

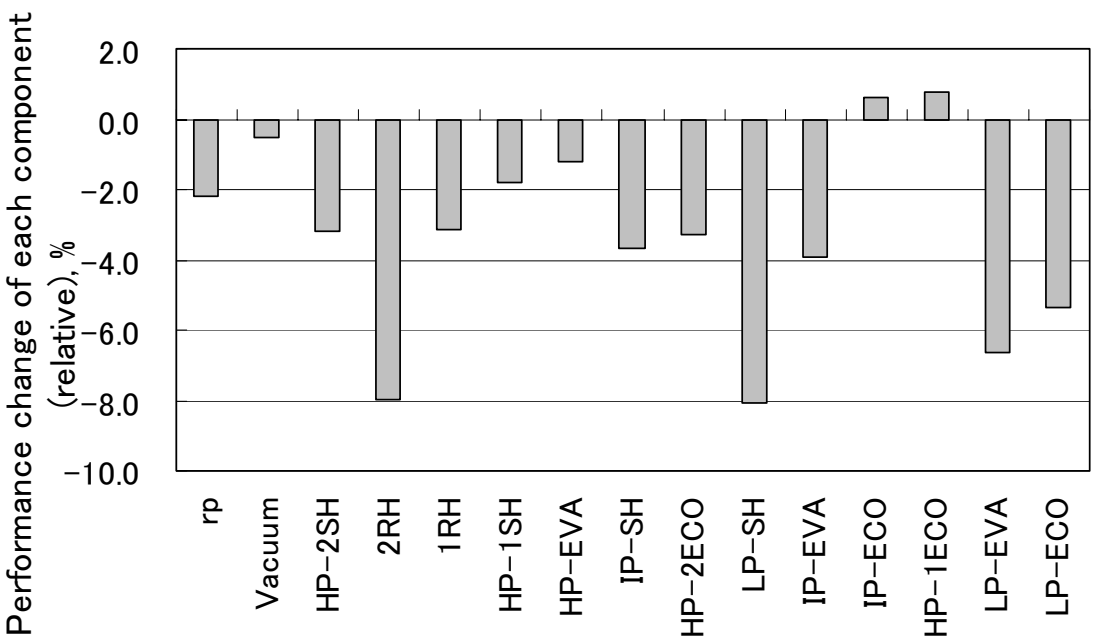

Fig. 13 Performance change of each component over about 4 years. 


\subsection{Quantitative estimation of effect of each deterioration factor on plant thermal efficiency}

In the previous section, the factors that affect thermal efficiency are examined in an actual 250MW-class GTCC power plant by heat balance analysis using operation data. In this section, the effects of these factors on thermal efficiency, that is, the factors causing thermal efficiency decrease are calculated quantitatively, and the factor with the largest effect on thermal efficiency is specified.

Each component performance and the operation conditions of the plant in the first performance test are changed into those in the fourth performance test conducted about 4 years after the first performance test. Then, the heat balance is analyzed on the basis of the component performance changes, and thermal efficiency is calculated. By this procedure, the effect of the changes in component performance on total thermal efficiency can be analyzed quantitatively.

The effects of each factor on thermal efficiency are shown in Fig 14. When all the values in this figure are added, they yield a total thermal efficiency change of $-1.4 \%$. From this figure, it is found that the main factor causing the thermal efficiency degeneration in this plant is the decrease in the adiabatic efficiency of ILPT, and that the decrease in the adiabatic efficiency of the compressor and condenser vacuum affect the plant thermal efficiency degeneration markedly. On the other hand, the decrease in the heat transfer coefficient of the heating surface at HRSG hardly affects the decrease in plant thermal efficiency.

In a past periodic inspection, the clearance in ILPT was enlarged to avoid contact between the casing and the blade; this corresponds well to the analytical result.

From the result, we could determine the factor that is the most effective for recovering the thermal efficiency of a plant and estimate the reduction in running cost due to fuel reduction by improving the factor, as well as the factor that has greatest effect on cost-effectiveness by comparing the repair cost with the reduction in running cost.

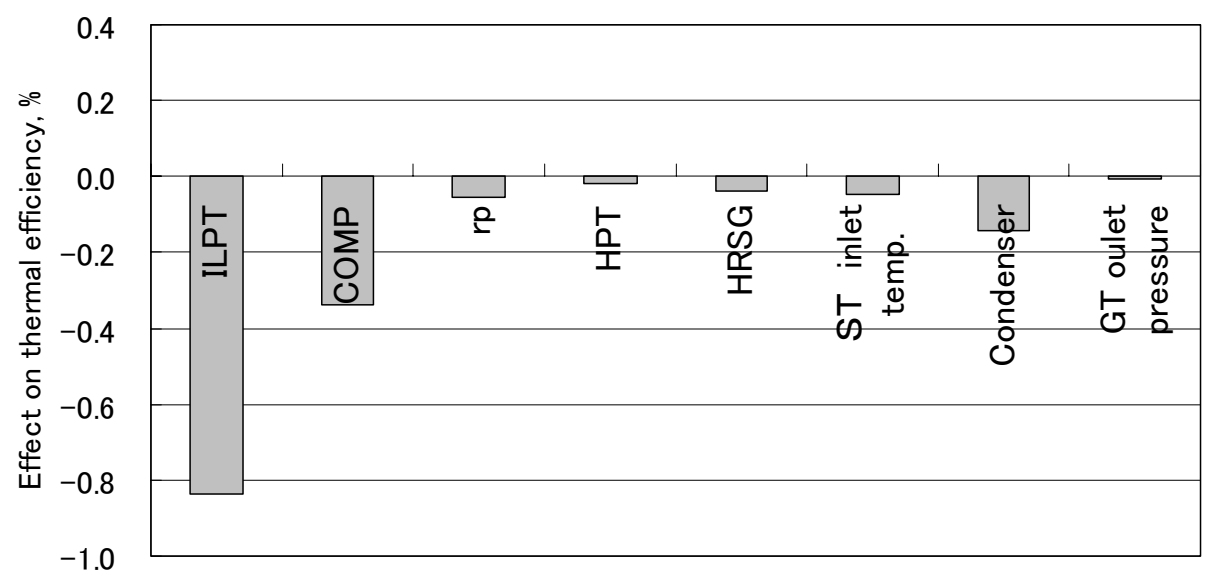

Fig. 14 Effects of each factor on thermal efficiency.

\section{Conclusions}

It is crucial to maintain or improve the thermal efficiency of actual GTCC power plants. Thus, the factors causing plant performance deterioration should be identified by clarifying the internal state of such plants.

Therefore, we developed a method based on heat balance analysis to calculate the immeasurable quantity of state and each component performance of GTCC power plants and convert them into corrected values using a performance function obtained from operation data, and moreover estimate quantitatively the effects of these factors on plant 
thermal efficiency.

By applying our method, an actual 250MW-class GTCC power plant was investigated to determine the performance degradation factors. As a result, it was confirmed that this method is very effective for investigating the main performance degradation factors.

It is hoped that this study will contribute to improvement in the thermal efficiency of GTCC power plants.

\section{References}

[1] Japanese Industrial Standard, Land Boilers - Heat Balancing, B 8222:1993.

[2] Japanese Industrial Standard, Steam Turbines - Acceptance Test, B 8102:2002

[3] Koda, E., Takahashi, T., "Method of system analysis, system analysis equipment using it and recording medium that can read computer that records system analysis program", Japanese Patent Disclosure 2001-101159 (2001)

[4] Koda, E., Takahashi, T., "Development of General Purpose Software to Analyze the Steady State of Power Generation Systems", Energy Conversion and Management Journal, Vol. 43 (1999), pp. 264-268.

[5] Takahashi, T., Koda, E., Mimaki, T., "A Systematic Analysis of the Effect of Air Humidification to Gas Turbine System", International Journal of the Japan Society of Mechanical Engineers, Series B, Vol. 45, No. 3 (2002), pp. 530-535.

[6] Koda, E., Takahashi, T., Uematsu, K., Yamashita. K., "Study on the High Efficiency Closed-Cycle Gas Turbine System for $\mathrm{CO}_{2}$ Collection", Journal of Gas Turbine Society of Japan, Vol. 30, No. 1 (2002), pp. 63-67.

[7] Takahashi, T., Koda, E., Nakao, Y., "Development of the Performance Deterioration Diagnosis Method to Thermal Power Plant Based on Heat and Mass Balance Analysis", International Conference on Power Engineering 2009, Vol. 2, (November 2009), Kobe, Japan, pp. 407-410.

[8] Thermal and Nuclear Power Engineering Society ed., Combined Generation (in Japanese), p. 31, Thermal and Nuclear Power Engineering Society.

[9] Umezawa, S., "Performance Diagnosis using Optical Torque Sensor for Selection of a Steam Supply Plant among Advanced Combined Cycle Power Plants", JSME Journal of Power and Energy Systems, Vol. 2 , No. 3 (2008), pp. 956-964.

[10] G. R. Wood, ASME Paper, 82-GT-20, 1982. 